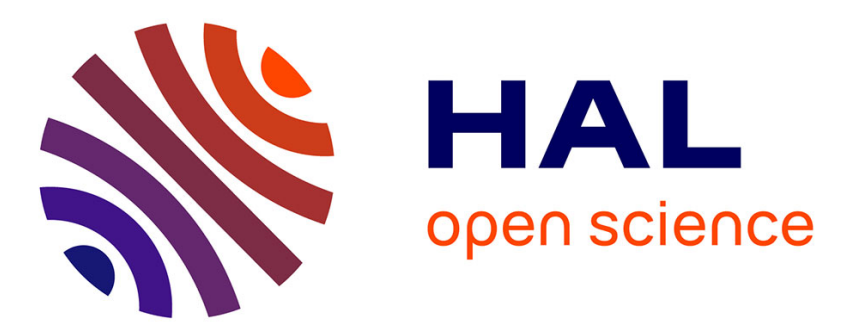

\title{
Soliton generation and rogue-wave like behavior through fourth order modulation instability
}

Kamal Hammani, Christophe Finot, Bertrand Kibler, Guy Millot

\section{To cite this version:}

Kamal Hammani, Christophe Finot, Bertrand Kibler, Guy Millot. Soliton generation and rogue-wave like behavior through fourth order modulation instability. Nonlinear Photonics, Jun 2010, Karlsruhe, Germany. pp.NMA6. hal-00469975

\section{HAL Id: hal-00469975 https://hal.science/hal-00469975}

Submitted on 29 Jul 2010

HAL is a multi-disciplinary open access archive for the deposit and dissemination of scientific research documents, whether they are published or not. The documents may come from teaching and research institutions in France or abroad, or from public or private research centers.
L'archive ouverte pluridisciplinaire HAL, est destinée au dépôt et à la diffusion de documents scientifiques de niveau recherche, publiés ou non, émanant des établissements d'enseignement et de recherche français ou étrangers, des laboratoires publics ou privés. 


\title{
Soliton generation and rogue-wave like behavior through fourth order modulation instabilty
}

\author{
Kamal Hammani,'Christophe Finot, Bertrand Kibler and Guy Millot \\ Laboratoire Interdisciplinaire Carnot de Bourgogne, UMR 5209 CNRS-Université de Bourgogne, Dijon, France \\ Christophe.Finot@u-bourgogne.fr
}

\begin{abstract}
We numerically study the dynamics of ultra-broadband wavelength converters based on fourth-order scalar modulation instability. We report the spontaneous emergence of solitons and trapped radiation waves as well as L-shaped associated statistical signatures.
\end{abstract}

OCIS codes: (060.4370) Nonlinear optics, fibers; (060.2320) Fiber optics amplifiers. (060.5530) Temporal solitons

\section{Introduction}

Optical fibers are convenient platforms with which to investigate a large set of fascinating fundamental nonlinear phenomena. Among the striking consequences of the interplay of dispersive and Kerr effects is the modulation instability (MI) [1], entailing the exponential growth of a weak perturbation at the expense of a continuous or quasicontinuous pump wave. MI is often associated with the anomalous dispersion regime where it has been intensively studied. However, MI is not restricted to this regime and the phase-matching condition required for the development of MI can also be fulfilled in normally dispersive fibers with an adequate fourth-order dispersion [2, 3]. This later configuration combined with the use of photonic crystal fibers has stimulated great attention, especially in view of the significant frequency shifts that can be achieved in wavelength conversion processes.

We focus here our attention on the spectro-temporal nature of both converted signal and idler. In particular, we report the spontaneous emergence of solitonic structures in the Stokes wave. Those solitons are then affected by the intrapulse Raman response of the fiber and further shift towards higher wavelengths. Regarding the anti-Stokes wave, an efficient optical trapping of dispersive waves mediated by solitons leads to spectral broadening towards shorter wavelengths. Furthermore, a statistical analysis of the peak-powers of both resulting signal and idler pulses highlights a 'rogue-wave' like behavior with pulses deviating strongly from the average behavior.

\section{Model, parameters and spectral properties}

In 2003, Harvey and coworkers have reported a new MI window by pumping with 70-ps 160-W pulses a photonic crystal fiber in the normal dispersion regime [3] (experimental spectra are recalled Fig. 1a1). The phase-matching conditions [2,3] enable to predict directly the wavelengths of the two narrow Stokes and anti-Stokes sidebands emerging far from the pump. However, if we look in more details into the experimental spectrum, we can notice that the resulting MI bands are much broader that what could be expected from the stringent phase-matching conditions.

More precisely, we can make out a small spectral peak located at the lower wavelength of the anti-Stokes wave as well as a significant broadening towards longer wavelengths of the Stokes. In order to provide a clear explanation, we numerically investigate the evolution of the optical wave in the fiber. The longitudinal evolution of envelope of the electrical field is accurately modeled by the generalized nonlinear Schrödinger equation [4]. Our numerical results plotted in Fig. 1a2 are in clear agreement with the previously published experimental results and reproduce the above mentioned spectral broadening of the Stokes and anti-Stokes waves.

\section{Spectro-temporal evolution and statistical distribution of the peak powers.}

In order to clarify the physical origin of the spectral evolution observed in normally dispersive fibers, we have computed the spectrogram of a unique shot at different propagation distances (Fig. 1b). The initial long-pulse is Fourier limited. At $50 \mathrm{~cm}$, two narrow Stokes and anti-Stokes sidebands develop, corresponding in the temporal domain to a strong modulation of the temporal profile. This modulation breaks the quasi-continuous pump to obtain fundamental solitons in the Stokes band which lies in the anomalous dispersion regime (Fig. 1b3). These optical structures are ultrashort (temporal duration below $100 \mathrm{fs}$ ), which explains the broadening of the spectral Stokes band. The intrapulse Raman response is then responsible for the progressive frequency shift (Figs. 1b4 towards higher wavelengths through the Raman soliton self-frequency shift effect [4]. Cross-phase modulation (XPM) in this multi-wavelength system was an additional key process taking part in the spectral expansion [4]. Regarding the evolution towards lower wavelengths of the anti-Stokes band, it is related to the phenomenon known as the radiation trapping by a soliton [4]. All those points are well illustrated in Fig. 1c which is a magnification of Fig. 1b4. An optical structure can be clearly distinguished at the wavelength of $1050 \mathrm{~nm}$. The details of the temporal intensity 
profile have been found in excellent agreement with the characteristic hyperbolic secant shape of a fundamental soliton. The associated trapped pulse in the normal dispersion regime temporally follows the soliton with a wavelength decreasing down to nearly $400 \mathrm{~nm}$.
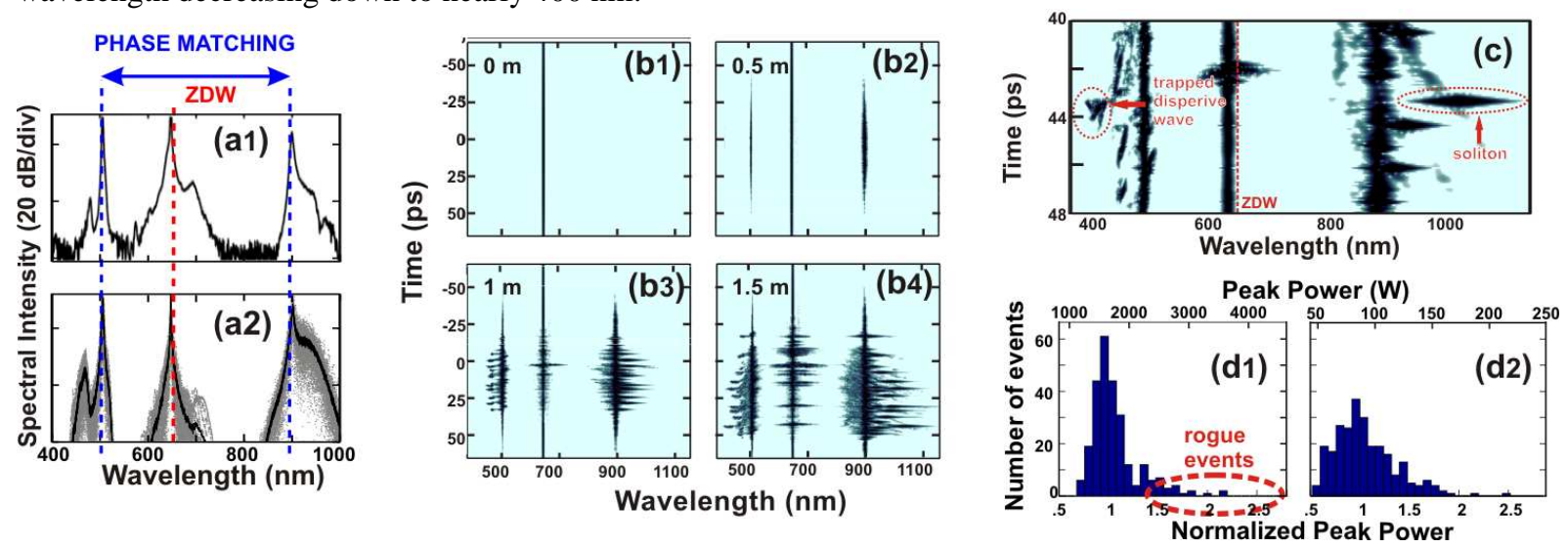

Fig. 1. The experimental spectrum recorded (a1) by Harvey et al. [3] is compared with our numerical results (a2) after $1 \mathrm{~m}$ of propagation. We have averaged (solid black line) our results over a set of 50 "shots" (grey dotted lines). (b) Spectro-temporal representations of optical field for various propagation lengths. $\begin{array}{ll}\text { (c) Magnification of 1(b4) between } 40 \text { and } 48 \mathrm{ps} & \text { (d) Statistical distribution of the peak powers for the }\end{array}$ soliton pulses in the Stokes band (d1) and dispersive trapped waves (d2) for a propagation length of $1.5 \mathrm{~m}$. Results are normalized relative to the median value (bottom) or expressed in Watts (top). 30 bins are used.

As already suggested by the optical spectra of Fig. 1a where significant changes are apparent, the obtained results differ markedly from shot to shot. Consequently, one may wonder if extreme-values statistics similar to the ones that have been reported in usual modulation instability based supercontinua can be recorded $[5,6]$. In order to measure the statistical distribution of the properties of the emerging optical structures, we have run 250 simulations in the presence of different random noise. Among the tens of solitons that emerge in the Stokes wave, we have systematically recorded the peak-power of the most intense pulse. Stokes wave and trapped dispersive waves have been conveniently isolated through an adequate spectral filtering. Note that, under XPM-induced modulation instability, the pump has also split into ultrashort structures and exhibits particularly apparent fluctuations (see Fig. 1a around $700 \mathrm{~nm}$ ).

Resulting probability distributions are summarized in Fig. 1d. At $1.5 \mathrm{~m}$, the distributions exhibit a tail towards high-power and the histogram exhibits the L-shape typical of extreme events [5] with several rare events presenting a peak power twice the median value. If solitons and trapped dispersive waves do not have exactly the same statistics, it is clear that their behaviors are intimately correlated, as recently suggested in [6].

\section{Conclusion}

In conclusion, we have carefully studied the process of wavelength conversion by fourth-order modulation instability. Our numerical results allow us to highlight the emergence of ultra-short fundamental solitons experiencing Raman self-frequency shift. This red-shift also induces a blue-shift of the anti-Stokes band indicating the presence of an optical radiation trapping by solitons which could be used to reach lower wavelengths. Moreover, a statistical analysis has revealed the emergence of rare but intense pulses that deviate strongly from the average behavior and from a Gaussian statistical distribution. [7]. All those points are crucial for the design of compact, stable, reliable and cost-efficient fiber format components for ultra broadband wavelength conversion.

\section{References}

1. K. Tai, A. Hasegawa, and A. Tomita, "Observation of modulational instability in optical fibers," Phys. Rev. Lett. 56, 135-138 (1986).

2. S. Pitois and G. Millot, "Experimental observation of a new modulational instability spectral window induced by fourth-order dispersion in a normally dispersive single-mode optical fiber," Opt. Commun. 226, 415-422 (2003).

3. J. D. Harvey, R. Leonhardt, S. Coen, G. K. L. Wong, J. C. Knight, W. J. Wadsworth, and J. S. Russell, "Scalar modulation instability in the normal dispersion regime by use of a photonic crystal fiber," Opt. Lett. 28, 2225-2227 (2003).

4. J. M. Dudley, G. Genty, and S. Coen, "Supercontinuum generation in photonic crystal fiber," Rev. Modern Physics 78, 1135-1184 (2006).

5. D. R. Solli, C. Ropers, P. Koonath, and B. Jalali, "Optical rogue waves," Nature 450, 1054 (2007).

6. M. Erkintalo, G. Genty, and J. M. Dudley, "Rogue wave like characteristics in femtosecond supercontinuum generation," Opt. Lett. 34, 24682470 (2009).

7. K. Hammani, C. Finot, B. Kibler, and G. Millot, "Soliton generation in a microstructured fiber by fourth order scalar modulation instability," IEEE Photon. J. 1, 205-212 (2009). 